\title{
MS03-P105 LATE | ContaMiner ANd ContaBase: Automated Identification of Unwantedly Crystallized Protein Contaminants
}

Hungler, Arnaud (King Abdullah University of Science and Technology, Thuwal, SAU); Momin, Afaque (King Abdullah University of Science and Technology, Thuwal, SAU); Diederichs, Kay (Universität Konstanz, Konstanz, GER); Arold, Stefan (Computational Bioscience Research Center (CBRC), King Abdullah University of Science and Technology, Thuwal, SAU)

Solving the phase problem in protein X-ray crystallography relies heavily on the identity of the crystallized protein, especially when molecular replacement (MR) methods are used. Yet, it is not uncommon that a contaminant crystallizes instead of the protein of interest. Such contaminants may be proteins from the expression host organism, protein fusion tags or proteins added during the purification steps. Many contaminants co-purify easily, crystallize and give good diffraction data. Identification of contaminant crystals may take time, since the presence of the contaminant is unexpected, and its identity unknown. We have established a webserver (ContaMiner) and a contaminant database (ContaBase), both available at strube.cbrc.kaust.edu.sa/contaminer/, to allow fast automated MR-based screening of crystallographic data against currently 80 known contaminants from several different expression systems and contaminating microbes. Here we present the latest developments of ContaMiner, and highlight a curious case of a structurally altered domain-swapped maltose-binding protein (MBP). 